\title{
RISK FACTORS OF INTERNET GAMING AMONG FILIPINO STUDENTS Marris R. Reyes
}

The Graduate School, University of Santo Tomas, España, Manila, Philippines and Extension and Training Director, Isabela State University - Jones Campus, Jones, Isabela Philippines. Email: marris.r.reyes@ @isu.edu.ph

\author{
Article History: Received on $22^{\text {nd }}$ April 2020, Revised on $30^{\text {th }}$ June 2020, Published on $17^{\text {th }}$ July 2020
}

\begin{abstract}
Purpose of the study: The main objective of this study is to examine the risk factors for internet gaming disorder (IGD). Specifically, it aims to: measure the severity of internet gaming among Filipino students; describe their internet gaming characteristic; and, identify which among the risk factors predict IGD.

Methodology: Students who displayed five or more symptoms in the Internet Gaming Disorder Scale - Short Form (IGD9-SF) were identified. The internet gaming characteristics, Brief Self Control Scale (BSCS) to identify self-control levels, and Mini-IPIP for the personality of the participants were also collected. Data were analyzed using descriptive statistics and linear regression analysis to identify the significant risk factors for IGD using SPSS 17.0.
\end{abstract}

Main Findings: Internet gaming characteristics such as time spent, money spent, and devices used were significant risk factors to IGD. Psychological factors such as self-control and personality traits (agreeableness and openness) were established and were also significant risk factors. The lower the self-control the higher the risk of developing IGD, as well as the more agreeable and open the gamers are the higher the risk to IGD.

Applications of this study: Researchers and therapists should focus on developing intervention methods focusing on minimizing the device's exposure to lessen time and money spent in gaming as well as strengthening the self-control of the gamers.

Novelty/Originality of this study: This psychological inquiry contributed to the emerging psychological disorder IGD shedding light on its current debates and controversies. This established risk factors to IGD among Filipino students who are time spent, money spent, devices used, self-control, and personality factors such as agreeableness and openness.

Keywords: Internet Gaming, Internet Gaming Disorder (IGD), Internet Gaming Characteristics, Internet Gamers, SelfControl, Personality Traits.

\section{INTRODUCTION}

Gaming is part of culture across the world (Kosheleva et al., 2020) and has an enormous impact on people's lives (King \& Delfabbro, 2019). Playing games is deemed an essential activity throughout human lives and most especially in child development (Rosyati et al., 2020). Rahmadi \& Prambayun, (2019) stressed that playing games are a vital part of human existence. The gaming industry, offline and online, plays a vital role in culture and economy (Liboriussen \& Martin, 2016). Generally, playing games are seen as valuable and positive to physical and mental health (King \& Delfabbro, 2019). On the other hand, modern gaming with the use of digital and internet technology, forming online gaming seen to be problematic.

The features of the internet and digital technology can be considered as one of the greatest human inventions. Its immense benefits to human lives especially when it comes to swift communication and industrial growth and development is indubitable. It has played an essential role in current pedagogical tools, especially in higher education (Karabaevna et al., 2020). It introduced recent economic approaches such as online marketing (Suriya, 2019) and new leisure activity such as video streaming and online video gaming (King \& Delfabbro, 2019; Pontes et al., 2019). However, behind its greatness lays huge detriments to human lives especially to mental health.

Esposito (2005) explained video games as "an interactive form of digital entertainment" which is designed to be played by a player. Video gaming with the use of the internet is called internet gaming which enables players to "alter their state of mind, experience emotions, satisfy psychological needs or simply pass time and escape reality" (Przybylski et al., 2016). It can also serve as a venue for socialization without face to face interaction, without going outside the school, work or play (Steinkuehler \& Williams, 2006) making it more engaging and addicting. For the past decade, internet gaming disorder (IGD) has been recognized as a global problem due to its damaging effects on the physical, psychological and social aspects of gamers - commonly children, adolescents, and young adults. In 2013, the American Psychiatric Association (APA) identified "Internet Gaming Disorder - IGD" in the emerging disorder of the "Diagnostic and Statistical Manual 5th Edition" (DSM-5) as a "condition worthy of future study" imploring for further studies (APA, 2013; Kuss, Griffiths \& Pontes 2017; Pontes \& Griffiths 2015).

Meanwhile, the World Health Organization (WHO, 2018) recognized and included it as a mental illness under addictive behaviours in the list of "mental, behavioral or neurodevelopmental disorder" of ICD-11. Primarily, the damaging effects of excessive internet gaming were related to its excessive time spent in gaming each day (Baggio, et al., 2016; King \& Delfabbro, 2018). Bax (2016) emphasized the vague conceptualization of IGD and viewed it as merely an issue of social deviance rather than a mental disorder. However, the extreme amount of time the gamers spent resulted in 
wasted opportunities, neglected social interactions, and essential interpersonal relationships, disregarding responsibilities (such as school or work), and poor eating habits and hygiene (King \& Delfabbro, 2018). WHO (2018) also emphasized that a gaming disorder changes the priorities of gamers leading to dramatically decreasing interest and capacity to reallife significant activities than gaming. Despite its recognition as a mental illness and the known seriousness of the condition; confusions, and debates on conceptualization and treatment among academicians, researchers, and practitioners.

APA (2013) defined IGD as "a condition where gamers play compulsively, to the exclusion of other interests, and their persistent and recurrent online activity results in clinically significant impairment or distress" ( as cited from King \& Delfabbro, 2019; Kuss et al., 2017; Pontes \& Griffiths, 2015) where they "experienced symptoms of withdrawal when kept away from gaming" (Braun et al., 2016). Studies suggest that when these individuals are engrossed in Internet games, specific pathways in their brains triggered in the same direct and intense way that a drug addict is affected by a particular substance (Braun et al., 2016; King \& Delfabbro, 2018; Kuss et al., 2017; Rho et al., 2017). Additionally, Zhang and Brand (2018)) explained that video games stimulate an extreme neurological reaction affecting pleasure and reward circuits of the brain, which is similar to a manifestation of an addicted behaviour response.

Moreover, González-Bueso et al. (2018) defined IGD as a persistent pattern of gaming behaviour including digital or video gaming (online or offline) which is manifested by "impaired control over gaming; increasing priority to gaming to the extent that gaming takes precedence over life interest and daily activities, and; continuation or escalation of gaming despite the occurrence of negative consequences". Various researches generated to provide a precise picture of the IGD and tools had been developed and tested to provide a clear diagnosis of the phenomenon. However, there is a scarce psychological inquiry to internet gaming characteristics such as time spent, the onset of gaming, money spent in gaming, gaming club, etc. which can be significant risk factors.

Additionally, researchers focused more on psychopathological risk factors such as depression, anxiety, autism, aggression, ADHD, and social phobia (Cole \& Hooley, 2013; Hyun et al., 2015; Mehroof \& Griffiths, 2010; Rho et al., 2017). This body of work assumes comorbidity of IGD to other psychological conditions. Possible risk factors such as personality and self-control of average gamers for gamers without such conditions needed further investigation.

Similar to other addiction and compulsive behaviour such as substance abuse, gambling, and sex, some individuals can be susceptible. The susceptibility can be due to risk factors including personal predispositions, psychological such as self-control and personality traits, unsafe environments, and the accessibility to online-gaming occasions and opportunities. Rho et al., (2017) listed possible risk factors among Korean participants such as personal profile, anxiety, depression, and stress considered to predict the likeliness of the occurrence of IGD. Further, Throuvala et al. (2019) concluded that personality traits and family factors among 225 young adults were predictors of IGD. Parental rejection and the importance of the father's role in upbringing are essential predictors of IGD (Throuvala et al., 2019). Another study done by Mehroof and Griffiths (2010) suggested that personality traits such as sensation seeking, anxiety, and neuroticism were closely linked with the onset of gaming behaviour, eventually leading to a problematic one. Similarly, traits like neuroticism, impulsivity, and agreeableness were found to be factors associated with IGD (Billieux et al., 2015; Braun et al., 2016). Nevertheless, Gervasi, et al. (2017) explained that these established relationships between personality traits and IGD are limited only to describing the casual link rather than knowing its effect on IGD. King \& Delfabbro (2018) also discussed the peer influences as a risk factor. Individuals who are surrounded by fellow video gamers are more likely to engage in increased online gaming (King et al., 2013). Thus, further analysis for examining its effect is essential to understand how personality factors affect the emergence of IGD.

Moreover, González-Bueso, et al. (2018) studied the link of parental psychoeducation to IGD. On the other hand, King and Delfabbro (2019) explained that "individuals from less stable and less warm families may be more at risk of problematic gaming and other online activities that enable an escape". Factors such as internet gaming characteristics, self-control, and personality traits as predictors of IGD needs further empirical evidence (Kuss et al., 2017). Moreover, understanding the risk factors of IGD can be essential for predicting those who are vulnerable to developing the condition. Knowing the risk factors is vital in designing treatment protocols and intervention programs addressing the challenging effects of IGD.

The enumerated characteristics, signs, and symptoms of this condition showed that it affects not only the mental health of an individual but their physical condition and sociological welfare as well. However, in the present day, the risk factors and causes are subject to further research and exploration. Notwithstanding the growing studies regarding the epidemiology of internet gaming disorder (IGD), predisposing factors were examined to a lesser extent such as personality traits and self-control. Thus, the main objective of this study is to investigate the risk factors for internet gaming disorder. Specifically, this study aims to: (1) measure the severity of internet gaming among Filipino College Students; (2) describe their internet gaming characteristic, and; (3) Identify which among the gaming characteristics, self-control, and personality profile are risk factors that predict IGD tendencies.

\section{RESEARCH METHODS}

The researchers identified three hundred fifty (350) college students from Isabela State University who have been playing video games particularly MMORPGs or MODAs for a year or more. Data were gathered manually using survey 
questionnaires composed of IGDS9-SF, Mini-IPIP, BSCS, and internet gaming characteristics checklist. Internet gaming characteristics profile by the nature of the research. Specific items were gaming devices used, the venue where the gamers were playing, the average time spent per week, and weekly expenses for video gaming.

The researcher used IGDS9-SF (Pontes \& Griffiths, 2015) to determine the Internet Gaming Disorder scores of the respondents. It is a valid and reliable short psychometric tool adapted from the nine core criteria that define IGD according to and in line with the DSM-5 (APA, 2013). IGDS9-SF is a tool used to describe and evaluate the severity of excessive internet gaming applicable not only for online but offline gaming as well. Pontes \& Griffiths (2015) expounded that a gamer that reacts five "very often" out of nine statements and is playing for a year or more can be diagnosed with IGD. Moreover, the tool is composed of nine questions that were answered using a 5-point Likert scale. The respondent was considered with Severe IGD symptoms if he answered 5 or more "very often" to the tool. For this study scoring was obtained by getting the sum of scores to range from 9 to 45 (Lemmens et al., 2015) with higher scores being indicative of higher degrees of gaming disorder tendencies (Pontes \& Griffiths, 2015). Scores were classified as: 9 to 18 ("low"); 19 to 27 ("moderate"); 28 to 36 ("high"); and 37 to 45 ("very high"). Note that the main purpose of this instrument is not to diagnose IGD but to assess its severity. Further, Cronbach's alpha is .801 .

To determine the personality traits and self-control of the respondents, the researcher utilized the Brief Self Control Scale (BSCS) and Mini-IPIP. Brief Self Control Scale by Tangey, Baumeister \& Boone (2004) is a 5-point Likert type scale measuring the level of self-control of an individual. Cronbach's alpha is .867. Also, Mini-IPIP by Donnellan et al. (2006) is a 20-item short form of the 50-item International Personality Item Pool - Five-factor Model measure (cited from Golberg, by Donnellan et. al, 2006). It is a five-point Likert scale with four items per Big Five Trait have consistent and acceptable internal consistencies with Cronbach's $\alpha=.860$.

Table 1: Description of Internet Gaming Characteristics and Psychological Personality

\begin{tabular}{llc}
\hline Variables & & $\begin{array}{c}\text { Number of } \\
\text { Items }\end{array}$ \\
\hline Internet Gaming Characteristics & $\begin{array}{l}\text { Money spent on gaming per week, Time Spent on Gaming } \\
\text { per week, Gaming Device, Gaming Venue }\end{array}$ & 4 \\
\hline Internet Gaming Disorder Scale & IGD Items based on DSM-5 & 9 \\
\hline Self-Control & Brief Self Control Scale (BSCS) & 13 \\
\hline Personality Factors & $\begin{array}{l}\text { Mini-IPIP (Neuroticism, Agreeableness, Extraversion, } \\
\text { Openness, Conscientiousness) }\end{array}$ & 20 \\
\hline
\end{tabular}

To determine the reliability of the instruments, the researcher used Reliability statistics Cronbach's alpha. Also, descriptive statistics, correctly mean scores, were used to identify the demographic characteristics, gaming characteristics, personality profile, self-control, and IGD scores of the respondents. Linear Regression analysis was utilized to determine which among the independent variables (demographic profile, gaming characteristics, and selfcontrol and personality traits) were risk factors to IGD. Data gathered was encoded and analyzed using Statistical Package for Social Sciences Version Seventeen (SPSS 17.0).

\section{RESULTS}

Interestingly, as seen in Table 2, most of the respondent gamers or 199 (56.9\%) spent 501-1000 pesos weekly for Internet Gaming; 93 (26.6\%) spent PHP 1001 to 1500; 55 (15.7\%) spent PHP 100-500 and only $3(0.9 \%)$ of them spent 1501 above. Most of the respondents $(135,38.6 \%)$ were spending an average of 49 to 72 hours weekly for online internet gaming; 123 (35.1\%) for 24 to 48 hours; 61 (17.4\%) for 1 to 24 hours; and 31 (8.9\%) game time weekly. Most of the respondents were using the mobile phone (146, 41.7\%); $132(37.7 \%)$ were using any possible devices; $38(10.9 \%)$ were consuming their own personal computers/laptop and 34 (9.7\%) were using electronic tablets. Meanwhile, most of the respondents play anywhere possible $(143,40.9 \%), 136$ (38.9\%) of them were playing at internet cafés, $55(15.7 \%)$ played at home and $16(4.6 \%)$ were playing at school. Alarmingly, most of the internet gamers spent most of their weekly allowance for internet games. Also, most gamers spent 2 to 4 days of their week for internet gaming which can result in detrimental physical and psychological health. This data reflects the poor monitoring skills of parents and other significant individuals to gamers.

Table 2: Internet Gaming Characteristics

\begin{tabular}{cccccc}
\hline $\begin{array}{c}\text { Internet Gaming } \\
\text { Characteristics }\end{array}$ & $\begin{array}{c}\text { Frequency } \\
(\mathbf{n = 3 5 0})\end{array}$ & Percentage & $\begin{array}{c}\text { Internet Gaming } \\
\text { Characteristics }\end{array}$ & $\begin{array}{c}\text { Frequency } \\
(\mathbf{n = 3 5 0})\end{array}$ & Percentage \\
\hline Money Spent & \multicolumn{5}{c}{ Device/Gadget Used } \\
\hline $100-500$ & 55 & 15.7 & Mobile Phone & 146 & 41.7 \\
\hline $501-1000$ & 199 & 56.9 & Tablet & 34 & 9.7 \\
\hline $1001-1500$ & 93 & 26.6 & Personal Computer & 38 & 10.9 \\
\hline 15001 above & 3 & .9 & Any possible Device & 132 & 37.7 \\
\hline Time Spent Weekly & & \multicolumn{5}{c}{ Venue of Gaming } \\
\hline
\end{tabular}




\begin{tabular}{cccccc}
\hline $1-24$ hours & 61 & 17.4 & Home & 55 & 15.7 \\
\hline $24-48$ hours & 123 & 35.1 & School & 16 & 4.6 \\
\hline $49-72$ hours & 135 & 38.6 & Internet Café & 136 & 38.9 \\
\hline 72 hours and above & 31 & 8.9 & Anywhere Possible & 143 & 40.9 \\
\hline
\end{tabular}

When it comes to IGD scores presented in table 3, most of the respondents scored moderate (164, 46.9\%), 66 (18.9\%) scored high, $61(17.4 \%)$ scored very high and 59 (16.9\%) scored low. Table 3 also revealed that most of the video gamer students have moderate scores. However, it is alarming that there were students who score high and very high. This means $17.4 \%$ of the college student gamers have IGD, which implies high prevalence. Further, this data inflicts that there were students who are already experiencing excessive and severe to internet gaming conditions.

Table 3: Internet Gaming Disorder Scores

\begin{tabular}{lll}
\hline IGD Score & Frequency $(\mathbf{n = 3 5 0})$ & Percentage $(\boldsymbol{\%})$ \\
\hline Low & 59 & 16.9 \\
\hline Moderate & 164 & 46.9 \\
\hline High & 66 & 18.9 \\
\hline Very High & 61 & 17.4 \\
\hline
\end{tabular}

Table 4 showed that device $(B=.062$, Beta=073), money spent $(B=.702$, $B$ eta $=471)$, and time spent $(B=.305, B e t a=.2467)$ weekly were highly significant internet gaming characteristics predictor of IGD tendencies. This data implies that higher investment in the game, the higher the risk factors of developing IGD. This data also infers that the higher the exposure thru time spent, the higher the potential of having severe internet addiction. And it suggests that the more devices used, the higher the tendency of having IGD.

Table 4: Internet Gaming Characteristics and IGD

\begin{tabular}{lllll}
\hline & Unstandardized Coefficients & Standardized Coefficients & Sig. & Collinearity Statistics \\
\hline & $\mathrm{B}$ & Beta & & VIF \\
\hline (Constant) & 2.150 & & .001 & \\
\hline Money Spent & .702 & .471 & .001 & 1.579 \\
\hline Time Spent & .305 & .247 & .001 & 1.421 \\
\hline Device & .062 & .073 & .018 & 1.099 \\
\hline Venue & .067 & .060 & .076 & 1.158 \\
\hline
\end{tabular}

Table 5 revealed that self-control $(\mathrm{B}=-.309$, Beta=-.269) was a highly significant risk factor to IGD tendencies. Among the big five personality factors, only openness $(B=-.212$, Beta=-.114) and agreeableness $(B=-.089$, Beta=-.063) were significant risk factors. This data implies that the lower the self-control of the gamers, the higher the risk factor to having IGD. The data also implies that lesser openness and lower agreeableness the higher the risk of developing IGD.

Table 5: Self-control, Personality Traits, and IGD

\begin{tabular}{ccccc}
\hline Personality Factors & Unstandardized Coefficients & Standardized Coefficients & Significance & $\begin{array}{c}\text { Collinearity } \\
\text { Statistics }\end{array}$ \\
\hline & $\mathrm{B}$ & Beta & VIF \\
\hline Constant & 2.150 & & .001 & .001 \\
\hline Self-control & -.309 & -.269 & .486 & 1.543 \\
\hline Neuroticism & .051 & .037 & .616 & 1.677 \\
\hline Extraversion & .048 & .034 & .033 & 1.011 \\
\hline Openness & -.212 & -.114 & .034 & 1.015 \\
\hline Agreeableness & -.089 & -.063 & .406 & 1.005 \\
\hline Conscientiousness & -.024 & -.832 & &
\end{tabular}

\section{DISCUSSION}

The researchers identified potential risk factors predicting IGD, precisely internet gaming characteristics, self-control, and personality traits. Further, the findings of this inquiry reflect that the majority of the student video gamers spent the majority amount of their allowance for internet gaming. This finding implies that most of the video gamers prioritize spending on online gaming rather than their necessities. Revealingly, it is alarming to know that students are spending an average of 3 to 4 days (72 to 96 hours) for internet gaming. This kind of behaviour entails decreased physical activities, sleep deprivation, absenteeism in school, and lesser time to socialize personally with their age groups. This behaviour may result in poor physical and mental health (King \& Delfabbro, 2019). As King and Delfabbro (2018) characterized that spending a significant amount of time in gaming leading to "missed opportunities and the interference with, and displacement of, normal routine and functioning, including basic activities (i.e., sleep, eating, and personal hygiene), real-world social interaction (i.e., talking to people, meeting friends face-to-face, and visiting family), and important 
responsibilities (i.e., school, work, and care of pets and children)". Neglecting other activities due to a deep interest in video gaming can lead to a considerable decrease in work- or education-related performance with their interpersonal and social relationship weaken or eventually end (Kirỏly et al, 2014). Gamers unknowingly sacrifice the essential events in their lives to excessively engage in the "virtual world" which resulted in detrimental effects in their lives.

Interestingly, although most of the respondents scored moderately in IGDS9-SF, it alarming to know that respondents with "very high" symptoms which means that IGD is prevalent to college students in the Philippines. This result contributed to the study of the prevalence of problematic gaming and IGD (Mihara \& Higuchi, 2017). Further, it indicates that student gamers have internet gaming disorder symptoms which can result in impaired physical, social and psychological functioning (APA, 2013).

Significantly, most of the items in internet gaming characteristics (money spent, time spent and device/gadgets used) were highly significant risk factors in predicting IGD. This finding implied that the more time spent in internet gaming the higher the risks in developing IGD. It also inferred the more elevated the money paid on video gaming, the higher the tendency to IGD. Availability and ease of access to gaming activities and devices essentially ruled the gaming behaviour of the gamers (Weis \& Cerankosky, 2010). Gamers who use mobile phones in gaming have a higher risk to cultivate IGD since the gamer access the game anytime and anywhere. This data is congruent with the recent findings that higher the money gamers invest in the game and time spent with it, the higher the risk of developing video gaming addiction (Rho et. al, 2017; Paik et. al, 2017). King and Delfabbro (2019) stated that "individuals with IGD often modify their environment to centralize gaming so that it is easier to initiate and maintain play". The finding in this research strengthens the argument that the more exposed the gamers in the internet gaming, the chance of severity in IGD becomes higher. And when they spent too much time, a high probability of paying their available resources (money) to continue playing. Another risk factor is the accessibility of the device, higher availability, the higher the opportunity to develop IGD. Collectively, gamers particularly those who were using devices such as computers and smartphones in gaming showed a greater prevalence of IGD (Paik et. al 2017). Gamers who spend most of their time in gaming is detrimental to their physical and psychological well-being (Kiròly, Griffiths \& Demetrovics, 2015). Nowadays, students have higher accessibility to the device since most of them have mobile phones in their hands with their internet access. Thus, excessive student allowance (money), exposure, and accessibility were significant risk factors in predicting IGD.

Examination of self-control and personality traits generated meaningful results. First, it indicated that the lesser selfcontrol of the gamers, the higher the risk factors in predicting IGD. Recent literature compared and contrast and proved the similarities of IGD to other addictions like gambling disorder, (Belanger-Lejars, 2015) and substance abuse (Yen et al., 2008). As explained by King and Delfabbro (2019), self-dysregulation can lead to developing behavioural addiction, such as IGD. In particular, self-control is an essential psychological factor affecting addiction (Tangey et al., 2004; Paik et. al, 2017; Maloney, Grawitch \& Barber, 2012). Lack of self-control is highly associated with internet addiction (Mei et al., 2016; Rho et al., 2017). Self-control encompasses a range of responses from overriding impulses to certain deliberate actions (Carver, 2005). As expounded by Tangey et al. (2004), individuals with higher self-control predict good adjustment skills, less pathology, better grades, and interpersonal success. Lack of self-regulation frequently has a higher risk of addictive activities (King \& Delfabbro, 2019). Lacking the ability to regulate oneself can lead to early experimentation with harmful activities leading to addictive behaviours (King \& Delfabbro, 2019). Individuals' inability to control oneself are more susceptible to be inclined to play games for longer time (Bailey, West \& Kuffel, 2013). Hence, individuals with lower self-control have an increased risk of developing IGD. It is beneficial for the therapist and related mental-health workers focusing on IGD to give attention and do something about the capacity of the client video gamer to self-control.

Second, personality traits openness, and agreeableness were other risk factors to IGD. Also, findings showed that the lesser openness tendencies gamers had, the higher risk factors. This data is consistent with the analysis of Wang, Ho, Chan \& Tse (2015) that low openness to experience has a significant link with IGD. The personality trait "openness" is one of the big five personality factors characterized by an individual's willingness to try new things, to be vulnerable, and the ability to think outside the box (Feist et al., 2018). It means that decreased willingness to try new things and to think differently from the norms is at the higher potentiality of developing IGD. This can be explained by the rules and features of different games in achieving goals and advancing at different levels. Most of the time video gamers are in the box of the game, were required to follow specific patterns, follow norms, and buy different products online to be successful in the game. Múller et al, (2014) stated that one explanation might be that gamers with low openness tend to stick to their gaming behaviour instead of exploring new activities. Hence, openness can be a vital risk factor for the conservation and development of IGD. Finally, findings implied that another significant risk factor is agreeableness. It is characterized by the individual's tendency to value benevolence, tradition, and conformity while avoiding placing too much importance on power, achievement, or pursuit of selfish pleasures (Feist et al., 2018). Lessened agreeableness directs a higher propensity to contend rather than cooperate. Video games require the high competitiveness of the video gamers to advance in different levels of the game (Kardefelt-Winther, 2017). Therefore, gamers can be addicted to the game since it stimulated their competitive nature to compete with other gamers. 


\section{CONCLUSION}

Internet gaming is prevalent among Filipino college students. Though most gamers seemed to have moderate symptoms (4 to 5 symptoms), the percentage of students with five or more symptoms - which can be a basis for IGD diagnosis was alarming. It was also interesting to know that most student gamers spend most of their time and allowance in onlinegaming which was supposed to be dedicated to studying.

The present study established that high exposure through time spent, money spent, the abundance of resources, and internet access to devices used in gaming are significant risk factors in the onset and eventually developing IGD. Therefore, parents, teachers, and other significant people of the students must be vigilant to the person experiencing or with characteristics of IGD. Their internet use must be monitored and encourage them to join healthy and socially engaging activities to lessen their preoccupation to internet gaming.

This study provided a direct link of IGD to self-control and strengthened the existing claim that restraint is a significant risk factor in emerging IGD symptoms. Hence, considering strengthening self-control of those internet gamers indeed is essential to eradicating, if not reducing their internet gaming. Personality traits openness and agreeableness were also significant risk factors that need further investigation.

\section{LIMITATION AND STUDY FORWARD}

Although this research has certain limitations, it generated valuable input to the existing knowledge to the understanding of risk factors for IGD specifically in terms of internet gaming characteristics, self-control, and personality traits. Establishing the IGD's effect on academic performance is an interesting inclusion for further research. Program developments and treatment methods should consider focusing on self-control, openness, and agreeableness for online gamers with IGD symptoms.

\section{ACKNOWLEDGEMENT}

This work was supported by the Commission on Higher Education (CHED) via CHED K to 12 Scholarship Program and polished by the valuable comments of Dr. Dante Garcia and Dr. Rhowel Dellosa.

\section{REFERENCES}

1. American Psychiatric Association. (2013). Diagnostic and statistical manual of mental disorders (DSM-5) (5th ed.). Washington, DC: American Psychiatric Association. https://doi.org/10.1176/appi.books.9780890425596

2. Bailey, K., West, R., \& Kuffel, J. (2013). What would my avatar do? Gaming, pathology, and risky decision making. Frontiers in psychology, 4, 609. https://doi.org/10.3389/fpsyg.2013.00609

3. Baggio, S., Dupuis, M., Studer, J., Spilka, S., Daeppen, J. B., Simon, O., Berchtold, A., \& Gmel, G. (2016). Reframing video gaming and Internet use addiction: Empirical cross-national comparison of heavy use over time and addiction scales among young users. Addiction, 111(3), 513-522. https://doi.org/10.1111/add.13192

4. Bax, T. (2016). "Internet Gaming Disorder" in China: Biomedical Sickness or Sociological Badness? Games and Culture, 11(3), 233-255. https://doi.org/10.1177/1555412014568188

5. Belanger-Lejars, V. (2015). Internet gaming disorder and gambling disorder: A comparison of individual factors. Journal of Psychology and Behavioral Science. SAGE Publication. 3(2) 122-140. https://doi.org/10.15640/jpbs.v3n2a12

6. Billieux, J., Schimmenti, A., Khazaal, Y., Maurage, P. \& Heeren, A. (2015). Are we psycho pathologizing everyday life? A tenable blueprint of behavioural addiction research. Journal of Behavioural Addiction 4(3), 119-123. https://doi.org/10.1556/2006.4.2015.009

7. Braun, B., Stopfer, J. M., Müller, K. W., Beutel, M. E., \& Egloff, B. (2016). "Personality and video gaming: Comparing regular gamers, non-gamers, and gaming addicts and differentiating between game genres". Computers in Human Behavior, 55(Part A), 406-412. https://doi.org/10.1016/j.chb.2015.09.041

8. Carver, C. S. (2005). "Impulse and constraint: perspectives from personality psychology, convergence with theory in other areas, and potential for integration". Personality and Social Psychology Review, 9(4), 312 333. https://doi.org/10.1207/s15327957pspr0904_2

9. Cole, S. H., \& Hooley, J. M. (2013). Clinical and personality correlate with MMO gaming: anxiety and absorption in problematic internet use. Social Science Computer Review, 31(4), 424436. https://doi.org/10.1177/0894439312475280

10. Day I. (2017). A family systems approach to the understanding and treatment of internet gaming disorder. The Family Journal: Counselling and Therapy for Couples and Families 25(3), 264-270. https://doi.org/10.1177/1066480717711108

11. Donnellan, M. B., Oswald, F. L., Baird, B. M., and Lucas, R. E. (2006). The Mini-IPIP scales: Tiny-yeteffective measure of the five factors of personality. Psychological Assessment, APA 18(2), pp. 192-203 https://doi.org/10.1037/1040-3590.18.2.192

12. Esposito, N. (2005). A short and simple definition of what a videogame is. Retrieved online http://summit.sfu.ca/item/258 
13. Feist, J., Feist, G. \& Roberts T. (2018). Theories of Personality: $9^{\text {th }}$ Edition. New York, McGraw-Hill Education.

14. Gervasi, A. M., La Marca, L., Constanzo, A., Pace, U., Guglielmucci, F. \& Schimmenti, A. (2017). Personality and internet gaming disorder: a systematic review of recent literature. Current Addiction Reports, 4, 293-307. https://doi.org/10.1007/s40429-017-0159-6

15. González-Bueso, V., Santamaría, J. J., Fernández, D., Merino, L., Montero, E., Jiménez-Murcia, S., Del PinoGutiérrez, A., \& Ribas, J. (2018). Internet Gaming Disorder in Adolescents: Personality, Psychopathology and Evaluation of a Psychological Intervention Combined With Parent Psychoeducation. Frontiers in psychology, 9, 787. https://doi.org/10.3389/fpsyg.2018.00787

16. Hyun, G. J., Han, D. H., Lee, Y. S., Kang, K. D., Yoo, S. K., Chung, U.-S., et al. (2015). Risk factors associated with online game addiction: a hierarchical model. Comput. Hum. Behav. 48, 706-713. https://doi.org/10.1016/j.chb.2015.02.008

17. Karabaevna, I., Riskulova, K., Ubaydullaevich, A. M., Yulduzkhon, I., Pulatova, T., \& Ravshanovna, N. (2020). The role of electronic pedagogical tools in higher education. Journal of Critical Review, 7 (5), 396-398. https://doi.org/10.31838/jcr.07.05.80

18. Kardefelt-Winther, D. (2014). Problematizing excessive online gaming and its psychological predictors. Computers in Human Behavior, 31, 118-122. https://doi.org/10.1016/j.chb.2013.10.017

19. Kardefelt-Winther, D. (2017), Conceptualizing Internet-use disorders: Addiction or coping process? Psychiatry Clinical Neuroscience, 71: 459-466. https://doi.org/10.1111/pcn.12413

20. King, D. \& Delfabbro, K. (2019). "Internet gaming disorder: theory, assessment, and treatment $1^{\text {st }}$ edition. Academic Press Elsevier. https://doi.org/10.1016/B978-0-12-812924-1.09986-X

21. King, D. L., and Delfabbro, P. H. (2018) Predatory monetization schemes in video games (e.g. 'loot boxes') and internet gaming disorder. Addiction, 113: 1967- 1969. https://doi.org/10.1111/add.14286

22. King, D. L., Delfabbro, P. H., Zwaans, T. \& Kaptasis, D. (2013). Clinical features and axis I comorbidity of Australian adolescent pathological Internet and video-game users. Australian and New Zealand Journal of Psychiatry, 47, 1058-1067. https://doi.org/10.1177/0004867413491159

23. Király, O., Griffiths, M. D. \& Demotrovics, Z. (2015). Internet gaming disorder and the DSM-5: conceptualization, debates, and controversies. Current Addiction Research 2, 254-262. https://doi.org/10.1007/s40429-015-0066-7

24. Király, O., Nagygyörgy, K., Griffiths, M. D., \& Demetrovics, Z. (2014). Problematic Online Gaming. In Behavioral Addictions: Criteria, Evidence, and Treatment (pp. 61-97). https://doi.org/10.1016/B978-0-12407724-9.00004-5

25. Kosheleva, A., Tamu Samedov, A. \& Sabirova, S. (2020). "Game as an activity in culture and pedagogy of Uzbekistan." Journal of Critical Reviews 7.4 (2020), 416-420. https://doi.org/10.31838/jcr.07.04.80

26. Kuss, D. J., Pontes, H., Király, O. \& Demetrovics, Z. (2018). A Psychological Overview of Gaming Disorder. The Oxford Handbook of Cyberpsychology, 1-23. https://doi.org/10.1093/oxfordhb/9780198812746.013.25

27. Kuss, D. J., Griffiths, M. D., \& Pontes, H. M. (2017). Chaos and confusion in DSM-5 diagnosis of Internet Gaming Disorder: Issues, concerns, and recommendations for clarity in the field. Journal of behavioural addictions, 6(2), 103-109. https://doi.org/10.1556/2006.5.2016.062

28. Lemmens, J. S., Valkenburg, P. M., \& Gentile, D. A. (2015). The Internet Gaming Disorder Scale. Psychological Assessment, 27(2), 567-582. https://doi.org/10.1037/pas0000062

29. Liboriussen, B., \& Martin, P. (2016). Special Issue: Games and Gaming in China. Games and Culture, 11(3), 227-232. https://doi.org/10.1177/1555412015615296

30. Maloney, P. W., Grawitch, M. J. \& Barber, L. K. (2012). The multi-factor structure of brief self-control scale: discriminant validity of restraint and impulsivity. Journal of Research in Personality. Elsevier. 46 (1), 111-115 https://doi.org/10.1016/j.jrp.2011.10.001

31. Mehroof, M. \& Griffiths, M. D. (2010). Online gaming addiction: the role of sensation seeking, self-control, neuroticism, aggression, state anxiety, trait anxiety. Cyberpsychology Behaviour and Social Networking, 13 (3), 313-316. https://doi.org/10.1089/cyber.2009.0229

32. Mei, S., Yau, Y. H., Chai, J., Guo, J., and Potenza, M. N. (2016). Problematic internet use, well-being, selfesteem, and self-control. Addiction Behaviour. 61, 74-79. https://doi.org/10.1016/j.addbeh.2016.05.009

33. Mihara, S. \& Higuchi, S. (2017). Cross-sectional and longitudinal epidemiological studies of Internet gaming disorder: a systematic review of the literature. Psychiatry and Clinical Neurosciences, https://doi.org/10.1111/pcn.12532

34. Müller, K. W., Beutel, M. Egloff, B., and Wölfling, K. (2014). Investigating Risk Factors for Internet Gaming Disorder: A Comparison of Patients with Addictive Gaming, Pathological Gamblers, and Healthy Controls regarding the Big Five Personality Traits. European Addiction Research 20, pp 129-136, https://doi.org/10.1159/000355832

35. Paik SH, Cho H, Chun JW, Jeong JE, Kim DJ (2017). Gaming device usage patterns predict internet gaming disorder: Comparison across Different Gaming Device Usage Patterns. Int J Environ Res Public Health. 2017;14(12):1512. https://doi.org/10.3390/ijerph14121512 
36. Pontes, H.M., Schivinski, B., Sindermann, C., Mei, L., Becker, B., Zhou, M. \& Montag, C. (2019). Measurement and Conceptualization of Gaming Disorder According to the World Health Organization Framework: the Development of the Gaming Disorder Test. Int $J$ Ment Health Addiction. https://doi.org/10.1007/s11469-019-00088-z

37. Pontes, H. M. (2016). Current practices in the clinical and psychometric assessment of internet gaming disorder in the era of the DSM-5: a mini-review of existing assessment tools. Mental Health and Addiction Research 1(1). https://doi.org/10.15761/MHAR.1000105

38. Pontes, H. M. \& Griffiths, M. D. (2015). Measuring DSM-5 internet gaming disorder: development and validation of a short psychometric scale. Computers in Human Behaviour 45 (2015) 137-143. https://doi.org/10.1016/j.chb.2014.12.006

39. Przybylski, A. K., Weinstein, N., \& Murayama, K. (2016). Internet gaming disorder: investigating the clinical relevance of a new phenomenon. American Journal of Psychiatry, 174, 230-236. https://doi.org/10.1176/appi.ajp.2016.16020224

40. Rahmadi L. \& Prambayun, A. (2019). Design of Game Design Document as an Interactive Media to Introduce the Culture of Pagar Alam City. Journal of Critical Reviews, 6 (5), 243-251.

41. Rho, M. J., Lee, H., Lee, T., Cho, H., Jung, D., Kim, D. \& Choi, Y. (2017). Risk factors for internet gaming disorder: psychological factors and internet gaming characteristics. International Journal of Environmental Research and Public Health 15, 40. https://doi.org/10.3390/ijerph15010040

42. Rosyati T., Purwanto, M., Gumelar, G., Yulianti, R., \& Mukharrom, T. (2020). "Effects of games and how parents overcome addiction to children." Journal of Critical Reviews 7(1), 65-67.

43. Steinkuehler, C.A. and Williams, D. (2006), Where Everybody Knows Your (Screen) Name: Online Games as "Third Places". Journal of Computer-Mediated Communication, 11: 885-909. https://doi.org/10.1111/j.10836101.2006.00300.x

44. Suriya, A. (2019). Psychology factor-based sentiment analysis for online product customer review using multimixed short text ridge analysis. Journal of Critical Reviews 6.6, 146-150.

45. Tangey, J. P., Baumeister, R. F. \& Boone, A. L. (2004). High Self-control predicts good adjustment, less pathology, better grades, and interpersonal success. Journal of Personality Psychology. 72, 271-324 https://doi.org/10.1111/j.0022-3506.2004.00263.x

46. Throuvala, M.A., Janikian M., Griffiths, M. D., Rennoldson, M. \& Kuss, D. (2019). The role of family and personality traits in Internet gaming disorder: A mediation model combining cognitive and attachment perspectives. Journal of Behavioral Addiction 8(4), 1-15. https://doi.org/10.1556/2006.8.2019.05

47. Wang, C. H., Ho, R. T., Chan, C. L. \& Tse, S. (2015). Prevalence and correlates of comorbid depression in a nonclinical online sample with DSM-5 internet gaming disorder. Journal of Affective Disorders, 226, 1-5. https://doi.org/10.1016/j.jad.2017.08.005

48. Weis, R., \& Cerankosky, B. C. (2010). Effects of video-game ownership on young boys' academic and behavioral functioning: a randomized, controlled study. Psychological Science, 21, 463-470. https://doi.org/10.1177/0956797610362670

49. World Health Organization (2018). Video Gaming Disorder. https://www.who.int/news-room/q-adetail/gaming-disorder

50. Yen, J. Y., Kho, C. H., Yen, C. F., Chen, S. H., Chung, W. L. \& Chen, C. C. (2008). Psychiatric symptoms in adolescents with internet addiction: Comparison with substance abuse. Journal of Psychiatry and Clinical Neurosciences, 62, pp. 9-16. https://doi.org/10.1111/j.1440-1819.2007.01770.x

51. Zhang, J.-T., \& Brand, M. (2018). Editorial: Neural Mechanisms Underlying Internet Gaming Disorder. Frontiers in Psychiatry, 9. https://doi.org/10.3389/fpsyt.2018.00404 